JOURNAL OF

FUNCTION SPACES AND APPLICATIONS

Volume 9, Number 3 (2011), 283-304 (c) 2011, Scientific Horizon http://www.jfsa.net

\title{
A Fubini Theorem in Riesz spaces for the Kurzweil-Henstock Integral ${ }^{1}$
}

\section{A. Boccuto, D. Candeloro and A. R. Sambucini}

(Communicated by Jürgen Appell)

2000 Mathematics Subject Classification. 28B15, 46G1.

Keywords and phrases. Riesz space, Kurzweil-Henstock integral, Fubini theorem.

Abstract. A Fubini-type theorem is proved, for the Kurzweil-Henstock integral of Riesz-space-valued functions defined on (not necessarily bounded) subrectangles of the "extended" real plane.

\section{Introduction}

The Fubini-Tonelli Theorem for the Kurzweil-Henstock integral is well known when the functions involved are defined on bounded $n$-dimensional intervals, see for example $[11,14,15,18,19,23]$ or [16] for unbounded intervals.

In general, its interest is motivated also by the fact that often simple or multiple integrals do not exist in the sense of Lebesgue, but only as improper ones, while they can be viewed as Kurzweil-Henstock integrals (see [5], [16]).

Moreover in applications involving stochastic processes and integration, it is advisable to work with mappings taking values in functional spaces, like $L^{1}$ or $L^{0}$ : more generally, we shall consider integration for Riesz spacevalued functions. In this context the Kurzweil-Henstock-type integral and its properties are investigated in $[3,4,6,7,10,12,21,29]$ and Fubini

\footnotetext{
${ }^{1}$ Supported by GNAMPA of CNR and University of Perugia
} 
Theorems in bounded $n$-dimensional intervals are obtained in [26, 27], and in a more general setting in [28]. In this paper we prove the following

Theorem. Let $J=H \times K \subset \widetilde{\mathbb{R}} \times \widetilde{\mathbb{R}}$ be a closed rectangle, $f: J \rightarrow R$ be $K H$-integrable and $w$-dominated, and suppose that the function

$$
Q(x)=\int_{K} f(x, y) d y
$$

is well-defined with respect to a common regulator in $H \backslash N$, where $N$ has Lebesgue measure zero. Then $Q$ is $K H$-integrable on $H$ and

$$
\int_{H}\left(\int_{K} f(x, y) d y\right) d x=\iint_{H \times K} f d x d y
$$

A crucial hypothesis in this theorem is the $w$-domination of $f$ (see Definition 3.7), related to the absolute $K H$-integrability of $f$ (see section 5).

\section{Preliminaries}

Let $\mathbb{N}, \mathbb{Q}, \mathbb{R}, \mathbb{R}^{+}, \widetilde{\mathbb{R}}$ be as usual. We often denote the elements of $\widetilde{\mathbb{R}}^{2}$ by the notations $\vec{t}$ or $(x, y)$. We begin with some preliminary definitions and results.

\section{Definitions 2.1.}

(2.1.1) A Riesz space $R$ is said to be Dedekind complete if every nonempty subset of $R$, bounded from above (below), has supremum (infimum) in $R$.

(2.1.2) A bounded double sequence $\left(a_{i, j}\right)_{i, j}$ in $R$ is called regulator or $(D)$ sequence if, for each $i \in \mathbb{N}, a_{i, j} \downarrow 0$, that is $a_{i, j} \geq a_{i, j+1}$ for all $j \in \mathbb{N}$ and $\bigwedge_{j \in \mathbb{N}} a_{i, j}=0$.

(2.1.3) Given a sequence $\left(x_{n}\right)_{n}$ in $R$, we say that $\left(x_{n}\right)_{n}$ (D)-converges to an element $x \in R$ if there exists a regulator $\left(a_{i, j}\right)_{i, j}$, satisfying the following condition:

for each mapping $\varphi \in \mathbb{N}^{\mathbb{N}}$, there exists an integer $n_{0}$ such that

$$
\left|x_{n}-x\right| \leq \bigvee_{i=1}^{\infty} a_{i, \varphi(i)}
$$


for all $n \geq n_{0}$. In this case, we write $(D) \lim _{n} x_{n}=x$ or simply $\lim _{n} x_{n}=x$, when no confusion can arise.

(2.1.4) We say that $R$ is weakly $\sigma$-distributive if for every regulator $\left(a_{i, j}\right)_{i, j}$ one has:

$$
\bigwedge_{\varphi \in \mathbb{N}}\left(\bigvee_{i=1}^{\infty} a_{i, \varphi(i)}\right)=0
$$

It is easy to check that the usual order convergence implies $(D)$ convergence, while the converse is true in weakly $\sigma$-distributive spaces (see [12]).

Throughout this paper, we shall always assume that $R$ is a Dedekind complete weakly $\sigma$-distributive Riesz space and that $J=H \times K$, where $H$ and $K$ are closed (not necessarily bounded) intervals of $\widetilde{\mathbb{R}}$.

Definitions 2.2. Let $\mathcal{C}=\left\{J^{*} \subset J, J^{*}\right.$ is a closed rectangle $\}$.

(2.2.1) Analogously to the one-dimensional case, a gauge on $J$ is a map $\delta$ defined on $J$ and taking values in the set of all open rectangles of $\widetilde{\mathbb{R}}^{2}$, such that $\vec{t} \in \delta(\vec{t})$ for every $\vec{t} \in J$ and $\delta(\vec{t})$ is bounded whenever $\vec{t} \in \mathbb{R}^{2}$.

(2.2.2) A division of $J$ is a finite collection $\Pi=\left\{W_{i}: i=1, \ldots, q\right\}$ of elements of $\mathcal{C}$ such that

(i): $\bigcup_{i=1}^{q} W_{i}=J$

(ii): $\lambda_{2}\left(W_{i} \cap W_{j}\right)=0$ whenever $i \neq j$.

A partition or tagged division of $J$ is a family $\Pi=\left\{\left(W_{i}, \vec{t}_{i}\right): i=\right.$ $1, \ldots, q\}$ satisfying (i), (ii) and such that:

(iii): $\vec{t}_{i} \in W_{i}, W_{i} \in \mathcal{C}, i=1, \ldots, q$.

A collection $\Pi=\left\{\left(W_{i}, \vec{t}_{i}\right): i=1, \ldots, q\right\}$ satisfying axioms (ii) and (iii), but not necessarily (i), is called decomposition of $J$. Given a gauge $\delta$, the partition or decomposition $\Pi$ is $\delta$-fine $(\Pi \prec \delta)$, if $W_{i} \subset \delta\left(\vec{t}_{i}\right)(i=1,2, \ldots, q)$.

(2.2.3) A division $\Pi$ is said to be cartesian if it is of the type $\Pi=$ $\left\{\left(\left[x_{k-1}, x_{k}\right] \times\left[y_{s-1}, y_{s}\right]: k=1, \ldots, n ; s=1, \ldots, l\right\},-\infty \leq \ldots<\right.$ $x_{k-1}<x_{k}<\ldots \leq+\infty,-\infty \leq \ldots<y_{s-1}<y_{s}<\ldots \leq+\infty$.

Let us denote by $\mathcal{C}_{c r}(J)$ the family of all cartesian divisions of $J$ whose real endpoints are rational.

In a similar way it is possible to define the cartesian partitions or decompositions of $J$.

Remark 2.3. Observe that every gauge $\delta$ on $J$ admits a $\delta$-fine partition (of $J$ ), see [16, Lemma 6.2.6] (the Cousin Lemma).

The following result will be useful in the sequel. 
Lemma 2.4. ([16, Lemma 6.6.2]) If $\vec{t}=(x, y)$, given a gauge $\delta(x, y)=$ $U_{1}(x, y) \times U_{2}(x, y)$, for any fixed $x \in H$ the mapping $\delta_{K, x}(y)=U_{2}(x, y)$ is a gauge on $K$. Analogously, for every $y \in K$, the map $\delta_{H, y}(x)=U_{1}(x, y)$ is a gauge on $H$. These two mappings are the projections of $\delta$. If the partitions

$$
\sigma(x)=\left\{\left(K_{i}(x), \eta_{i}(x)\right): i=1, \ldots, q(x)\right\}
$$

are $\delta_{K, x}$-fine for every $x \in H$, then there exists a gauge $\Delta$ on $H$, such that, whenever $\pi_{H}=\left\{\left(H_{j}, \xi_{j}\right): j=1, \ldots, m\right\}$ is a $\Delta$-fine partition of $H$, then the associated partition

$$
\left\{\left(H_{j} \times K_{i}\left(\xi_{j}\right),\left(\xi_{j}, \eta_{i}\left(\xi_{j}\right)\right)\right): j=1, \ldots, m, i=1, \ldots, q\left(\xi_{j}\right)\right\}
$$

is $\delta$-fine.

A partition of this type (see Figure 1) is also called a compound partition $\left(\left[\begin{array}{ll}16, \S & 6.2\end{array}\right]\right)$.

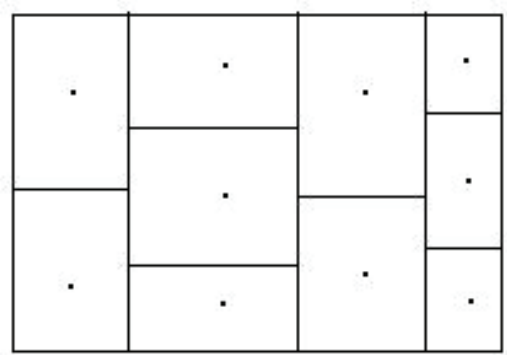

FIGURE 1. compound partition

\section{The Kurzweil-Henstock integral}

The aim of this section is to introduce the Kurzweil-Henstock integral for Riesz space-valued functions, defined in a possibly unbounded subrectangle of $\widetilde{\mathbb{R}}^{2}$. Given a measurable set $E \subset \widetilde{\mathbb{R}}\left(\widetilde{\mathbb{R}}^{2}\right)$, we denote by $|E|=\lambda_{1}(E)$ $\left(\lambda_{2}(E)\right)$ its one-(two)-dimensional Lebesgue measure (this quantity can be finite or not).

We now recall the concept of "one-dimensional" Kurzweil-Henstock integral.

Definition 3.1. ([3]) Let $(a, b)$ be a (possibly unbounded) subinterval of the extended real line. A function $f:(a, b) \rightarrow R$ is said to be KurzweilHenstock integrable (briefly, $K H$-integrable) on $(a, b)$ if there exist $I \in R$ 
and a regulator $\left(a_{i, j}\right)_{i, j}$ such that to every $\varphi \in \mathbb{N}^{\mathbb{N}}$ there corresponds a gauge $\gamma:(a, b) \rightarrow\{C \subset \widetilde{\mathbb{R}}: C$ open subinterval $\}$, such that $\gamma(x)$ is bounded whenever $x \in(a, b)$, and

$$
\left|\sum_{\Pi} f\left(\xi_{k}\right)\right| I_{k}|-I| \leq \bigvee_{i=1}^{\infty} a_{i, \varphi(i)}
$$

whenever $\Pi=\left\{\left(I_{k}, \xi_{k}\right), k=1, \ldots, p\right\}$ is a $\gamma$-fine partition of $(a, b)$ and in the involved sum only the terms for which $\left|I_{k}\right|<+\infty$ are included. If $f$ is $K H$ - integrable we write $\int_{a}^{b} f=I$.

The two-dimensional integral can be defined in a similar way. Given any partition $\Pi=\left\{\left(W_{i}, \vec{t}_{i}\right), i=1, \ldots, q\right\}$ of $J$ and a function $f: J \rightarrow R$, we call Riemann sum of $f$ (and we write $\sum_{\Pi} f$ ) the quantity $\sum_{i=1}^{q} f\left(\overrightarrow{t_{i}}\right) \lambda_{2}\left(W_{i}\right)$, where in the sum only the terms for which $W_{i}$ is a bounded rectangle are included.

The definition then becomes:

Definition 3.2. (Particular case of [4, Definition 3.1]) We say that a function $f: J \rightarrow R$ is Kurzweil-Henstock integrable (in short $K H$-integrable ) on $J$ if there exist an element $I \in R$ and a regulator $\left(a_{i, j}\right)_{i, j}$ in $R$ such that for every $\varphi \in \mathbb{N}^{\mathbb{N}}$ there corresponds a gauge $\delta$ such that

$$
\left|\sum_{\Pi} f-I\right| \leq \bigvee_{i=1}^{\infty} a_{i, \varphi(i)}
$$

whenever $\Pi=\left\{\left(W_{i}, \vec{t}_{i}\right), i=1, \ldots, q\right\}$ is a $\delta$-fine partition of $J$. In this case we say that $I$ is the $K H$-integral of $f$, and denote the element $I$ by the symbol $\int_{J} f$.

It is easy to see that $I$ is uniquely determined.

Since the definition of the $K H$-integral is given as a suitable limit, then

Proposition 3.3. (Bolzano-Cauchy condition, see [4, Theorem 3.4]) $A$ map $f: J \rightarrow R$ is $K H$-integrable if and only if there exists a regulator $\left(a_{i, j}\right)_{i, j}$ such that

(3.3.1) for all $\varphi \in \mathbb{N}^{\mathbb{N}}$ there is a gauge $\delta$ such that for all $\delta$-fine partitions $\Pi_{1}, \Pi_{2}$ of $J$ we have

$$
\left|\sum_{\Pi_{1}} f-\sum_{\Pi_{2}} f\right| \leq \bigvee_{i=1}^{\infty} a_{i, \varphi(i)} .
$$


As a consequence of [4], if $f$ is $K H$-integrable on $J$, then $f$ is $K H$ integrable on each set $E \in \mathcal{C}, E \subset J$. Moreover

Proposition 3.4. If $E \in \mathcal{C}, E_{i} \in \mathcal{C}, i=1, \ldots, n$, and $E=\cup_{i=1}^{n} E_{i}$, $\lambda_{2}\left(E_{i} \cap E_{j}\right)=0$ whenever $i \neq j$ and $f$ is $K H$-integrable on $E_{i}$ for every $i$, then $f$ is $K H$-integrable on $E$, and

$$
\int_{E} f=\sum_{i=1}^{n} \int_{E_{i}} f
$$

We now turn to the main properties of this two-dimensional $K H$-integral.

Theorem 3.5. (Henstock Lemma) Let $f: J \rightarrow R$ be a $K H$-integrable function, and fix any $(D)$-sequence $\left(a_{i, j}\right)_{i, j}$, such that to every $\varphi: \mathbb{N} \rightarrow \mathbb{N}$ there corresponds a gauge $\delta$ such that

$$
\left|\int_{J} f-\sum_{\Pi} f\right| \leq \bigvee_{l=1}^{\infty} a_{l, \varphi(l)}
$$

for every partition $\Pi \prec \delta$. If $\Pi=\left\{\left(W_{i}, \vec{t}_{i}\right): i=1, \ldots, r\right\} \prec \delta$, then for every $L \neq \emptyset, L \subset\{1, \ldots, r\}$, we have:

$$
\begin{aligned}
& \left|\sum_{i \in L} f\left(\vec{t}_{i}\right) \lambda_{2}\left(W_{i}\right)-\sum_{i \in L} \int_{W_{i}} f\right| \leq \bigvee_{l=1}^{\infty} a_{l, \varphi(l)} \\
& \sum_{i=1}^{r}\left|f\left(\vec{t}_{i}\right) \lambda_{2}\left(W_{i}\right)-\int_{W_{i}} f\right| \leq 2 \bigvee_{l=1}^{\infty} a_{l, \varphi(l)}
\end{aligned}
$$

Proof. The proof of (3.5.1) is contained in [4, Theorem 4.2]. While for (3.5.2), the technique is inspired by the one used in [21] and [22]. By the Maeda-Ogasawara-Vulikh representation theorem (see [1]), there exists a compact Hausdorff extremely disconnected topological space $\Omega$ such that $R$ can be embedded as a solid subspace of $C_{\infty}(\Omega)=\{f: \Omega \rightarrow \widetilde{\mathbb{R}}:$ $f$ is continuous, and $\{\omega \in \Omega:|f(\omega)|=+\infty\}$ is nowhere dense in $\Omega\}$. By virtue of (3.5.1), for any partition $\Pi=\left\{\left(W_{i}, \vec{t}_{i}\right): i=1, \ldots, s\right\} \prec \delta, \omega \in \Omega$ and $L \subset\{1, \ldots, s\}$ we get:

$$
0 \leq\left|\sum_{i \in L} f\left(\vec{t}_{i}\right) \lambda_{2}\left(W_{i}\right)-\sum_{i \in L} \int_{W_{i}} f\right|(\omega) \leq\left[\bigvee_{l=1}^{\infty} a_{l, \varphi(l)}\right](\omega) \quad \text { for all } \omega \in \Omega .
$$

Fix now $\omega \in \Omega$. If $\left[\bigvee_{l=1}^{\infty} a_{l, \varphi(l)}\right](\omega)=+\infty$, then there is nothing to prove. 
Suppose that $\left[\bigvee_{l=1}^{\infty} a_{l, \varphi(l)}\right](\omega) \in \mathbb{R}$, and let $L$ (resp. $\left.L^{\prime}\right)$ be the set of all indexes $i \in\{1, \ldots, s\}$ such that

$$
\left\{\sum_{i \in L}\left[f\left(\vec{t}_{i}\right) \lambda_{2}\left(W_{i}\right)-\int_{W_{i}} f\right]\right\}(\omega) \geq 0 \quad[\text { resp. }<0] .
$$

We have:

$$
\begin{aligned}
& 0 \leq \sum_{i=1}^{s}\left|f\left(\overrightarrow{t_{i}}\right) \lambda_{2}\left(W_{i}\right)-\int_{W_{i}} f\right|(\omega)=\sum_{i \in L}\left[f\left(\vec{t}_{i}\right) \lambda_{2}\left(W_{i}\right)-\int_{W_{i}} f\right](\omega)+ \\
& -\sum_{i \in L^{\prime}}\left[f\left(\vec{t}_{i}\right) \lambda_{2}\left(W_{i}\right)-\int_{W_{i}} f\right](\omega) \leq 2\left[\bigvee_{l=1}^{\infty} a_{l, \varphi(l)}\right](\omega) .
\end{aligned}
$$

From this the assertion follows.

The proof of the next proposition is straightforward.

Proposition 3.6. The integral is a linear positive functional. Moreover, if both $f$ and $|f|$ are $K H$-integrable on $J$, then $\left|\int_{J} f\right| \leq \int_{J}|f|$.

Definition 3.7. We say that a function $f: J \rightarrow R$ is $w$-dominated if there exist a $K H$-integrable map $\widehat{h}: J \rightarrow \mathbb{R}^{+}$and a positive element $w \in R$, such that $|f(\vec{t})| \leq \widehat{h}(\vec{t}) w$ for all $\vec{t} \in J$.

Remark 3.8. One of the motivations for this definition is that it implies that almost null functions have null integral. In the real-valued case every function vanishing almost everywhere is $K H$-integrable and its integral on $J$ is null, while in a Riesz space $R$, this is not true in general. For example, let $R$ be the Riesz space of all eventually null real sequences, and $\left(u_{n}\right)_{n}$ be the canonical basis. It is easy to check that the function $f:[0,1] \rightarrow R$, defined by $f=\sum_{n=1}^{\infty} u_{n} \chi_{\{1 / n\}}$, is not $K H$-integrable (see [2]). Observe moreover that $w$-domination is related to $K H$-integrability of $|f|$, for more details see section 5 .

The $K H$-integral turns out to be the natural one for all simple functions. More precisely, as a particular case of [4, Theorem 3.7], we have the following

Theorem 3.9. Let $x \in R, E$ be any Borel subset of $J$, with $\lambda_{2}(E)<$ $+\infty$, and $g$ defined by $g(\vec{t})=\chi_{E}(\vec{t}) x$. Then $g$ is $K H$-integrable, and

$$
\int_{J} g=x \lambda_{2}(E)
$$


Theorem 3.10. (Lebesgue dominated convergence theorem, see [4, Theorem 4.5]) Let $\left(f_{n}: J \rightarrow R\right)_{n}$ be a sequence of integrable functions, and assume that $\kappa: J \rightarrow R$ is an integrable map, such that $\left|f_{n}(\vec{t})\right| \leq \kappa(\vec{t})$ for all $\vec{t} \in J$ and $n \in \mathbb{N}$. Suppose that:

(3.10.1) there are a function $f: J \rightarrow R$, a $K H$-integrable map $\widehat{h}: J \rightarrow \mathbb{R}^{+}$ and a regulator $\left(a_{i, j}\right)_{i, j}$ such that to every $\varphi \in \mathbb{N} \mathbb{N}$ and $\vec{t} \in J$ there corresponds an integer $p=p(\vec{t})$ such that, for every $n \geq p(\vec{t})$,

$$
\left|f_{n}(\vec{t})-f(\vec{t})\right| \leq \widehat{h}(\vec{t})\left(\bigvee_{i=1}^{\infty} a_{i, \varphi(i)}\right) .
$$

Then $f$ is integrable on $J$ and $\int_{J} f=\lim _{n} \int_{J} f_{n}$.

Before applying Theorem 3.10 we introduce the following definitions (see [27, Definitions 4 and 5]):

Definition 3.11. We say that the function $f: J \rightarrow R$ is continuous with respect to a common regulator on $J$ if there exist a regulator $\left(a_{i, j}\right)_{i, j}$ in $R$ and a $K H$-integrable function $\widehat{h}: J \rightarrow \mathbb{R}^{+}$with the following property: for every $\varphi \in \mathbb{N}^{\mathbb{N}}$ and $\vec{t} \in J$ there exists an open interval $J_{\vec{t}} \subset \mathbb{R}^{2}$ containing $\vec{t}$ such that, whenever $\vec{t} \in J_{\vec{t}} \cap J, \quad|f(\vec{t})-f(\vec{t})| \leq \widehat{h}(\vec{t})\left(\bigvee_{i=1}^{\infty} a_{i, \varphi(i)}\right)$.

Definition 3.12. A sequence $\left(f_{n}\right)_{n}$ converges to $f$ on $J$ with respect to a common regulator if there are a $K H$-integrable function $\widehat{h}: J \rightarrow \mathbb{R}^{+}$ and a regulator $\left(a_{i, j}\right)_{i, j}$ in $R$ with the following property: for all $\varphi \in \mathbb{N}^{\mathbb{N}}$ and for every $\vec{t} \in J$ there is $p(\vec{t}) \in \mathbb{N}$ such that, for any $n \geq p(\vec{t})$, $\left|f_{n}(\vec{t})-f(\vec{t})\right| \leq \widehat{h}(\vec{t})\left(\bigvee_{i=1}^{\infty} a_{i, \varphi(i)}\right)$.

Remark 3.13. Note that, when $R=\mathbb{R}$ and $H=K=\widetilde{\mathbb{R}}$, Definitions 3.11 and 3.12 reduce to the classical continuity and pointwise convergence respectively: indeed, it is sufficient to take the function $\widehat{h}$ defined by: $\widehat{h}(\vec{t})=e^{-|\vec{t}|^{2}} \chi_{\mathbb{R}^{2}}(\vec{t})$.

Lemma 3.14. If $f: J \rightarrow R$ is continuous with respect to a common regulator and $f \geq 0$, then there is a sequence of positive step functions $\left(s_{k}\right)_{k}$ such that $s_{k} \uparrow f$ on $J$ with respect to the same common regulator.

Proof. At the generic $k$-th step, $k \in \mathbb{N}$, we split the set $J_{k}:=$ $J \cap([-k, k] \times[-k, k])$, if it is nonempty, into pairwise disjoint intervals with diameter smaller than $1 / k$. Denote this collection of subintervals of $J$ by $D_{k}=\left\{E_{i}^{k}: i=1,2, \ldots, m_{k}\right\}$. Take the sequence $\left(D_{k}\right)_{k}$ in such a way that $D_{k+1}$ is a refinement of $D_{k}$ for all $k$ (that is, for every $E_{i}^{k+1}$ there 
is $E_{j}^{k}$ with $E_{i}^{k+1} \subset E_{j}^{k}$ unless $\left.E_{i}^{k+1} \subset J_{k+1} \backslash J_{k}\right)$. For each fixed $k \in \mathbb{N}$, when $\vec{t} \in J_{k}$ there is a unique element $E_{i}^{k}$ with $\vec{t} \in E_{i}^{k}$. For $\vec{t} \in J_{k}$ set

$$
s_{k}(\vec{t})=\inf \left\{f(\vec{t}): \vec{t} \in E_{i}^{k}\right\} \text { for } \vec{t} \in E_{i}^{k}\left(i=1,2, \ldots, m_{k}\right) .
$$

Of course $s_{k}(\vec{t}) \leq s_{k+1}(\vec{t})$ for every $\vec{t} \in E$ and $k \in \mathbb{N}$. We show that $s_{k}(\vec{t}) \uparrow f(\vec{t})$ for every $\vec{t} \in J$. For all $\vec{t} \in J$ there is $k_{0}=k_{0}(\vec{t}) \in \mathbb{N}$ with $\vec{t} \in J_{k}$ whenever $k \geq k_{0}$. Since $f$ is continuous with respect to a common regulator, there is a regulator $\left(a_{i, j}\right)_{i, j}$ in $R$ such that for any $\varphi \in \mathbb{N}^{\mathbb{N}}$ we can find an open interval $J_{\vec{t}}$ with $\vec{t} \in J_{\vec{t}}$ and $|f(\vec{t})-f(\vec{t})| \leq$ $\widehat{h}(\vec{t})\left(\bigvee_{i=1}^{\infty} a_{i, \varphi(i)}\right)$ whenever $\vec{t} \in J_{\vec{t}}$. There is $p(\vec{t}) \in \mathbb{N}$, greater than $k_{0}(\vec{t})$, such that for every $k \geq p(\vec{t})$

$$
\left|s_{k}(\vec{t})-f(\vec{t})\right| \leq \widehat{h}(\vec{t})\left(\bigvee_{i=1}^{\infty} a_{i, \varphi(i)}\right) .
$$

This concludes the proof.

\section{The Fubini theorem}

The aim of this section is to prove a version of the Fubini theorem, adapting some techniques due to [16, 26, 27]. We begin introducing the following definition about iterated integrals.

Definition 4.1. Let $f: J \rightarrow R$, and $S \subset H$. We say that $Q(x)=$ $\int_{K} f(x, y) d y$ exists with respect to a common regulator for each $x \in S$ if there are a $K H$-integrable function $h^{*}: H \rightarrow \mathbb{R}^{+}$(with respect to the onedimensional Lebesgue measure) and a regulator $\left(b_{i, j}\right)_{i, j}$ with the property that to each $x \in S$ and $\varphi \in \mathbb{N}^{\mathbb{N}}$ there corresponds a gauge $\delta_{x}$ on $K$ with

$$
\left|Q(x)-\sum_{i=1}^{q(x)} f\left(x, \eta_{i}(x)\right)\right| K_{i}(x)|| \leq h^{*}(x)\left(\bigvee_{i=1}^{\infty} b_{i, \varphi(i)}\right)
$$

whenever $\left\{\left(K_{i}(x), \eta_{i}(x)\right): i=1, \ldots, q(x)\right\}$ is a $\delta_{x}$-fine partition.

Remark 4.2. Observe that, when $R=\mathbb{R}$ and $H=K=\widetilde{\mathbb{R}}$, by [16, Lemma 6.6.1], if $f$ is $K H$-integrable on $J$, then $Q(x)=\int_{K} f(x, y) d y$ is defined $\lambda_{1}$-almost everywhere. Moreover, in the real case, the function $Q$, if it exists, always satisfies condition (1) of Definition 4.1 (replacing double sequences with $\varepsilon>0$ and suitably choosing null sets, if needed; in this case we can consider $h^{*}$ similarly as in Remark 3.13). 
Using this definition we are able to formulate this version of the Fubini Theorem which allows us to calculate the integral of $f$ via the one of $Q$.

Theorem 4.3. (Fubini Theorem) Let $f: J \rightarrow R$ be $K H$-integrable and $w$-dominated. Assume further that a $\lambda_{1}$-null set $N$ exists, such that $Q(x)=\int_{K} f(x, y) d y$ exists with respect to a common regulator for each $x \in H \backslash N$. Then, setting $Q(x)=0$ for every $x \in N$, we have that $Q$ is $K H$-integrable on $H$ and

$$
\int_{H}\left(\int_{K} f(x, y) d y\right) d x=\iint_{H \times K} f .
$$

Proof. Put $M_{0}=N \times K$. Then $\lambda_{2}\left(M_{0}\right)=0$ and by virtue of bounded integrability we get $\int_{J}|f| 1_{M_{0}}=0$. By this and Theorem 3.5 there are two regulators $\left(a_{i, j}\right)_{i, j},\left(b_{i, j}\right)_{i, j}$, such that for every $\varphi \in \mathbb{N}^{\mathbb{N}}$ a gauge $\delta$ on $J$ can be found, with

$$
\left|\sum_{l=1}^{q} f\left(\zeta_{l}\right) \lambda_{2}\left(Z_{l}\right)-\sum_{l=1}^{q} \int_{Z_{l}} f\right| \leq \bigvee_{i=1}^{\infty} a_{i, \varphi(i)}
$$

whenever $\Pi=\left\{\left(Z_{l}, \zeta_{l}\right): l=1, \ldots, q\right\}$ is a $\delta$-fine partition of $J$, and

$$
\sum_{p=1}^{s}\left|f\left(\omega_{p}\right)\right| \lambda_{2}\left(Y_{p}\right) \leq \bigvee_{i=1}^{\infty} b_{i, \varphi(i)}
$$

whenever $\Pi^{*}=\left\{\left(Y_{p}, \omega_{p}\right): \omega_{p} \in M_{0}, p=1, \ldots, s\right\}$ is a $\delta$-fine decomposition of $J$. Let now $\left(c_{i, j}\right)_{i, j}$ be a regulator and $h^{*}$ be a map, according to (1). Thanks to $K H$-integrability of $h^{*}$ on $H$, there exist a gauge $\Delta_{0}$ on $H$ and a positive constant $M$ with the property that

$$
\sum_{\pi_{0}} h^{*}=\sum_{\pi_{0}}\left|V_{j}\right| h^{*}\left(v_{j}\right) \leq M
$$

for every $\Delta_{0}$-fine partition $\pi_{0}$ of $H, \pi_{0}=\left\{\left(V_{j}, v_{j}\right): j=1, \ldots, s\right\}$.

Let now $\vec{t}=(x, y)$. For $x \in H \backslash N$, let $\sigma(x)=\left\{\left(K_{i}(x), \eta_{i}(x)\right): i=\right.$ $1, \ldots, q(x)\}$ be a $\delta_{K, x}$-fine partition for which

$$
\left|Q(x)-\sum_{i=1}^{q(x)} f\left(x, \eta_{i}(x)\right)\right| K_{i}(x)|| \leq h^{*}(x)\left(\bigvee_{i=1}^{\infty} c_{i, \varphi(i)}\right) ;
$$

and for any $x \in N$ let $\sigma(x)$ be a $\delta_{K, x}$-fine partition. Let $\Delta$ be a gauge on $H$ according to Lemma 2.4. Without loss of generality, we can choose $\Delta$ such that $\Delta(x) \subset \Delta_{0}(x)$ for every $x \in H$. Pick a $\Delta$-fine partition $\pi_{H}=$ 
$\left\{\left(H_{j}, \xi_{j}\right): j=1, \ldots, m\right\}$ of $H$, and let $\pi=\left\{\left(H_{j} \times K_{i}\left(\xi_{j}\right),\left(\xi_{j}, \eta_{i}\left(\xi_{j}\right)\right)\right)\right.$ : $\left.j=1, \ldots, m, i=1, \ldots, q\left(\xi_{j}\right)\right\}$ be a compound partition. Since $f$ is $w$ dominated then $Q$ can be extended to all of $H$ putting $Q(x)=0$ on $N$, so we have

$$
\begin{aligned}
\left|\sum_{\pi_{H}} Q\left(\xi_{j}\right)\right| H_{j}\left|-\int_{H \times K} f\right| \leq \mid & \sum_{\pi_{H}, \xi_{j} \in H \backslash N}\left|H_{j}\right|\left[Q\left(\xi_{j}\right)-\sum_{\sigma\left(\xi_{j}\right)} f\left(\xi_{j}, \eta_{i}\left(\xi_{j}\right)\right)\left|K_{i}\left(\xi_{j}\right)\right|\right] \mid \\
& +\left|\sum_{\pi_{H}, \xi_{j} \in N}\right| H_{j}\left|\left[Q\left(\xi_{j}\right)-\sum_{\sigma\left(\xi_{j}\right)} f\left(\xi_{j}, \eta_{i}\left(\xi_{j}\right)\right)\left|K_{i}\left(\xi_{j}\right)\right|\right]\right| \\
& +\left|\sum_{\pi} f\left(\xi_{j}, \eta_{i}\left(\xi_{j}\right)\right)\right| H_{j}|| K_{i}\left(\xi_{j}\right)\left|-\sum_{\pi} \int_{H_{j} \times K_{i}\left(\xi_{j}\right)} f\right| \\
\leq & \left|\sum_{\pi_{H}, \xi_{j} \in H \backslash N}\right| H_{j}\left|\left[Q\left(\xi_{j}\right)-\sum_{\sigma\left(\xi_{j}\right)} f\left(\xi_{j}, \eta_{i}\left(\xi_{j}\right)\right)\left|K_{i}\left(\xi_{j}\right)\right|\right]\right| \\
& +\sum_{\pi_{H}, \xi_{j} \in N}\left|f\left(\xi_{j}, \eta_{i}\left(\xi_{j}\right)\right)\right|\left|H_{j}\right|\left|K_{i}\left(\xi_{j}\right)\right| \\
& +\left|\sum_{\pi} f\left(\xi_{j}, \eta_{i}\left(\xi_{j}\right)\right)\right| H_{j}|| K_{i}\left(\xi_{j}\right)\left|-\sum_{\pi} \int_{H_{j} \times K_{i}\left(\xi_{j}\right)} f\right| \\
\leq & \sum_{\pi_{H}, \xi_{j} \in H \backslash N}^{\infty}\left|H_{j}\right| h^{*}\left(\xi_{j}\right)\left(\bigvee_{i=1}^{\infty} c_{i, \varphi(i)}\right)+\bigvee_{i=1}^{\infty} b_{i, \varphi(i)}+\bigvee_{i=1}^{\infty} a_{i, \varphi(i)} \\
\leq & M\left(\bigvee_{i=1}^{\infty} c_{i, \varphi(i)}\right)+\bigvee_{i=1}^{\infty} b_{i, \varphi(i)}+\bigvee_{i=1}^{\infty} a_{i, \varphi(i)} .
\end{aligned}
$$

This concludes the proof.

Remark 4.4. Because of the symmetry between $x$ and $y$, we can obtain also the analogous result for the other iterated integral. Moreover, in Theorem 4.3 we can replace the hypothesis of $w$-domination of $f$ assuming that the sets $N$ and $M_{0}=N \times K$ are negligible (i.e. such that the integral of every function which is zero outside $N$ and $M_{0}$ respectively is zero, see $[16, \S 2.5$ and 6.3$])$. Observe also that the equivalence between the negligibility and $\lambda_{2}$-null sets is automatically satisfied when $f$ is bounded (and integrable).

As a consequence of the Fubini Theorem we obtain:

Corollary 4.5. Let $f: J \rightarrow R$ be $w$-dominated and continuous with respect to a common regulator on $J$. Then 
(4.5.1) $f(x, \cdot)$ is $K H$-integrable on $K$ for almost all $x \in H$ and $Q(x)=\int_{K} f(x, y) d y$ exists with respect to a common regulator almost everywhere in $H$;

(4.5.2) $Q$ is $K H$-integrable almost everywhere on $H$;

(4.5.3) $f$ is $K H$-integrable on $J$ and

$$
\int_{J} f(x, y) d x d y=\int_{H}\left(\int_{K} f(x, y) d y\right) d x .
$$

Proof. First of all note that, proceeding analogously as in [27], it is easy to check that the assertion follows if $f$ is a step function, defined in a compact interval of $J$.

Let us turn to the general case. Suppose first that $f \geq 0$, and let $\left(s_{k}\right)_{k}$ be as in Lemma 3.14. Note that $s_{k}(\vec{t}) \uparrow f(\vec{t})$ with respect to a common regulator. By virtue of the Lebesgue dominated Convergence Theorem 3.10, $f$ is integrable on $J$ and

$$
\int_{J} f(\vec{t}) d t=\lim _{k} \int_{J} s_{k}(\vec{t}) d t
$$

Observe now that $s_{k}(x, \cdot)$ is $K H$-integrable on $K, s_{k}(x, \cdot) \uparrow f(x, \cdot)$ and $f(x, \cdot)$ is continuous for every $x \in H$ with respect to a common regulator (independent on $x$ ). By construction, the function

$$
Q_{k}(x):=\int_{K} s_{k}(x, y) d y
$$

is also $K H$-integrable on $K$ for all $x \in H$.

Let $\widehat{h}$ be as in Definition 3.7. Since $\widehat{h}$ is real-valued, by [16, Lemma 6.6.1] there is a set $N \subset K$, such that for all $x \in H \backslash N$ the function $\widehat{h}(x, \cdot)$ is integrable on $K$. We have that for each $x \in H \backslash N$ the sequence $\left(\int_{k} s_{k}(x, y) d y\right)_{k}$ is bounded, with bound independent on $x \in H \backslash N$ and depending only on the regulator $\left(a_{i, j}\right)_{i, j}$ associated with continuity of $f(x, \cdot), w$ and $\widehat{h}$ as in Definition 3.7. Indeed, for any choice of $\varepsilon>0$ and $\varphi \in \mathbb{N}^{\mathbb{N}}$, for $k$ large enough and $m, n \geq k$ we get

$$
\left|\int_{J \backslash J_{k}}\left(s_{n}-s_{m}\right)\right| \leq 2\left(\int_{J \backslash J_{k}} \widehat{h}\right) w \leq \varepsilon w
$$

thanks to the absolute continuity of the Kurzweil-Henstock integral (see [16, $\S 6.5]$ ). Moreover, by virtue of continuity of $f$ with respect to the same 
regulator,

$$
\left|\int_{J_{k}}\left(s_{n}-s_{m}\right)\right| \leq 2\left(\int_{J_{k}} \widehat{h}\right)\left(\bigvee_{i=1}^{\infty} a_{i, \varphi(i)}\right) .
$$

Thus we can apply the Lebesgue dominated Convergence Theorem 3.10, getting that for every $x \in H \backslash N$ the function $f(x, \cdot)$ is $K H$-integrable on $K$ and

$$
Q(x):=\int_{K} f(x, y) d y=\lim _{k} Q_{k}(x), \quad x \in H \backslash N
$$

with respect to the same regulator, independent on $x$. The other properties follow from this and Theorem 4.3. Finally, if $f$ is not of a constant sign, the assertion follows by linearity, considering $f^{+}$and $f^{-}$.

\section{5. w-domination and absolute $K H$-integrability}

Now we compare the condition of $w$-domination with absolute $K H$ integrability. For this aim we introduce some concepts on rectangle functions following the approach of $[9,25]$. We shall assume that $f$ is $K H$-integrable and $w$-dominated. Let $q: \mathcal{C} \rightarrow R$ be the rectangle function defined by $q(W):=\left|\int_{W} f\right|$. Then $q$ is subadditive, namely for any two divisions $\mathcal{W}=\left\{W_{i}\right\}_{i=1}^{n}, \mathcal{Z}=\left\{Z_{j}\right\}_{j=1}^{l}$ of $J$, such that $\mathcal{Z}$ is a refinement of $\mathcal{W}$, we have $\sum_{i=1}^{n} q\left(W_{i}\right) \leq \sum_{j=1}^{l} q\left(Z_{j}\right)$.

We want to apply to $q$ the theory given in [8]. So we say that:

Definition 5.1. A rectangle function $q$ is $(C)$-integrable if

(5.1.1) there is a regulator $\left(a_{i, j}\right)_{i, j}$ with the property that to every $\varphi \in \mathbb{N}^{\mathbb{N}}$ there correspond an element $I(q) \in R$ and a division $\Pi_{\varphi}$ such that, for each division $\Pi$ which refines $\Pi_{\varphi}$,

$$
\left|I(q)-\sum_{L \in \Pi} q(L)\right| \leq \bigvee_{i=1}^{\infty} a_{i, \varphi(i)} .
$$

Moreover

Lemma 5.2. $q$ is $(C)$-integrable if and only if

(5.2.1) there exists a regulator $\left(a_{i, j}\right)_{i, j}$ with the property that for any $\varphi \in \mathbb{N}^{\mathbb{N}}$ there is a division $\Pi_{\varphi}$ such that for all divisions $\Pi$, $\Pi^{\prime}$ which refine $\Pi_{\varphi}$,

$$
\left|\sum_{L \in \Pi} q(L)-\sum_{M \in \Pi^{\prime}} q(M)\right| \leq \bigvee_{i=1}^{\infty} a_{i, \varphi(i)} .
$$


Using these facts we have first that:

Lemma 5.3. If $f$ is an $R$-valued, $w$-dominated and $K H$-integrable function, and

$(\mathbb{E})(f):=\sup \left\{\sum_{i}\left|\int_{W_{i}} f\right|:\left\{W_{i}\right\}_{i=1}^{n}\right.$ is a division of $\left.J, W_{i} \in \mathcal{C}\right\} \in R$,

then $q$ is $(C)$-integrable and $I(q)=V(f)$.

Proof. Observe that, since $f$ is integrable on every subrectangle of $J$, it makes sense to define the supremum in (4). Moreover, by [16, Theorem 6.5.1], $V(\widehat{h})<+\infty$ since $\widehat{h}$ is $K H$-integrable.

Step 1. We prove that $V(f) \in R$, namely that the involved supremum is finite. By virtue of $w$-domination, for every $\vec{t} \in J$, we get $|f(\vec{t})| \leq \widehat{h}(\vec{t}) w$. Taking the integrals of $f$ and $-f$, we obtain

$$
q(W)=\left|\int_{W} f\right| \leq\left[\int_{W} \widehat{h}\right] w=: \widehat{q}(W) w
$$

for every $W \in \mathcal{C}$, and hence $q(W) \leq \widehat{q}(W) w$. Thus for any division $\left\{W_{i}\right\}_{i=1}^{n}$ of $J$ we obtain

$$
\sum_{i=1}^{n} q\left(W_{i}\right) \leq\left[\sum_{i=1}^{n} \widehat{q}\left(W_{i}\right)\right] w \leq V(\widehat{h}) w \in R .
$$

Taking the suprema, it follows that $V(f) \in R$.

Step 2. We now prove that the supremum in (4) can be obtained with a countable family of divisions. First of all observe that $q$ is subadditive while $\widehat{q}$ is additive and $q(\cdot) \leq \widehat{q}(\cdot) w$. Let $\mathcal{A}$ be any division of $J$ and denote by $\mathcal{A}^{\prime}$ the cartesian division generated by $\mathcal{A}$. For the sake of simplicity we shall assume $J=\widetilde{\mathbb{R}}^{2}$, and denote

$$
\mathcal{A}^{\prime}=\left\{\left(a_{i}, a_{i+1}\right) \times\left(b_{k}, b_{k+1}\right): i=0, \ldots, n ; k=0, \ldots, l\right\},
$$

where $a_{0}=-\infty, a_{n+1}=+\infty, b_{0}=-\infty, b_{l+1}=+\infty$. Of course $\mathcal{A}^{\prime}$ is finer than $\mathcal{A}$, so $S\left(\mathcal{A}^{\prime}\right) \geq S(\mathcal{A})$, where $S(\Pi)=\sum_{W \in \Pi} q(W)$ for any division $\Pi$.

Fix arbitrarily $\varepsilon>0$. We construct a new cartesian division $\mathcal{A}^{\prime \prime}$ as follows: by absolute continuity, there exist rational finite numbers $\alpha_{0}, \alpha_{\infty}, \beta_{0}, \beta_{\infty}$ such that $\alpha_{0}<a_{1}, \beta_{0}<b_{1}, \alpha_{\infty}>a_{n}, \beta_{\infty}>b_{l}$ and such that

$$
\sum_{i} \int_{H_{i}} \widehat{h} \leq \frac{\varepsilon}{2}
$$


for any decomposition $\left\{H_{i}\right\}$ of $J$ with rectangles, disjoint from ]$\alpha_{0}, \alpha_{\infty}[\times] \beta_{0}, \beta_{\infty}[$. So define

$$
\begin{aligned}
\mathcal{A}^{\prime \prime}= & \left\{\left(a_{i}^{\prime \prime}, a_{i+1}^{\prime \prime}\right) \times\left(b_{k}^{\prime \prime}, b_{k+1}^{\prime \prime}\right)\right\}, \text { where }\left\{a_{i}^{\prime \prime}\right\}_{i}=\left\{-\infty, \alpha_{0}, a_{1}, a_{2}, \ldots, a_{n}, \alpha_{\infty},+\infty\right\}, \\
& \left\{b_{k}^{\prime \prime}\right\}_{k}=\left\{-\infty, \beta_{0}, b_{1}, b_{2}, \ldots, b_{l}, \beta_{\infty},+\infty\right\} .
\end{aligned}
$$

Then clearly $\mathcal{A}^{\prime \prime}$ refines $\mathcal{A}^{\prime}$. We now will construct a rational cartesian division $\mathcal{B}$ as follows: to each point $a_{i}, i=1, \ldots, n$ and $b_{k}, k=1, \ldots l$, we associate two rational points $\alpha_{i}^{\prime}, \alpha_{i}^{\prime \prime}, \beta_{k}^{\prime}, \beta_{k}^{\prime \prime}$, such that $\alpha_{i}^{\prime}<a_{i}<\alpha_{i}^{\prime \prime}$, $\beta_{k}^{\prime}<b_{k}<\beta_{k}^{\prime \prime}, \alpha_{0}<\alpha_{1}^{\prime}, \alpha_{\infty}>\alpha_{n}^{\prime \prime}, \beta_{0}<\beta_{1}^{\prime}, \beta_{\infty}>\beta_{l}^{\prime \prime}$, and

$$
\begin{aligned}
& \max \left\{\left(\alpha_{i}^{\prime \prime}-\alpha_{i}^{\prime}\right)\left(\beta_{k+1}^{\prime}-\beta_{k}^{\prime \prime}\right),\left(\alpha_{i+1}^{\prime}-\alpha_{i}^{\prime \prime}\right)\left(\beta_{k}^{\prime \prime}-\beta_{k}^{\prime}\right),\left(\alpha_{i}^{\prime \prime}-\alpha_{i}^{\prime}\right)\left(\beta_{k}^{\prime \prime}-\beta_{k}^{\prime}\right)\right\} \\
\text { (宦 }) & \eta\left(\frac{\varepsilon}{64(n+3)(l+3)}\right) .
\end{aligned}
$$

(Here, given $\sigma>0, \eta(\sigma)$ is that positive real number related to absolute continuity of $\int \widehat{h}$.) Then the construction of $\mathcal{B}$ is obtained by taking all intervals $\left(\widetilde{a}_{r} \widetilde{a}_{r+1}\right) \times\left(\widetilde{b}_{s}, \widetilde{b}_{s+1}\right)$, where $\widetilde{a}_{r}=$ $\tilde{\mathrm{b}}_{s} \infty, \alpha_{0}, \alpha_{1}^{\prime}, \alpha_{1}^{\prime \prime}, \alpha_{2}^{\prime}, \alpha_{2}^{\prime \prime} \ldots, \alpha_{n}^{\prime}, \alpha_{n}^{\prime \prime}, \alpha_{\infty},+\infty$ $\widetilde{b}_{s}=-\infty, \beta_{0}, \beta_{1}^{\prime}, \beta_{1}^{\prime \prime}, \beta_{2}^{\prime}, \beta_{2}^{\prime \prime} \ldots, \beta_{l}^{\prime}, \beta_{l}^{\prime \prime}, \beta_{\infty},+\infty$. Hence, the unbounded rectangles of $\mathcal{B}$ are the same as for $\mathcal{A}^{\prime \prime}$. We now compare $S(\mathcal{B})$ with $S\left(\mathcal{A}^{\prime \prime}\right)$, simply considering the bounded rectangles. It is readily seen that to each "typical" rectangle of $\mathcal{A}^{\prime \prime},\left(a_{i}^{\prime \prime}, a_{i+1}^{\prime \prime}\right) \times\left(b_{k}^{\prime \prime}, b_{k+1}^{\prime \prime}\right)$, there correspond (at most) nine rectangles from $\mathcal{B}$, i.e.

$$
\begin{array}{ccc}
\left(\alpha_{i}^{\prime}, \alpha_{i}^{\prime \prime}\right) \times\left(\beta_{k}^{\prime}, \beta_{k}^{\prime \prime}\right), & \left(\alpha_{i}^{\prime \prime}, \alpha_{i+1}^{\prime}\right) \times\left(\beta_{k}^{\prime}, \beta_{k}^{\prime \prime}\right), & \left(\alpha_{i+1}^{\prime}, \alpha_{i+1}^{\prime \prime}\right) \times\left(\beta_{k}^{\prime}, \beta_{k}^{\prime \prime}\right), \\
\left(\alpha_{i}^{\prime}, \alpha_{i}^{\prime \prime}\right) \times\left(\beta_{k}^{\prime \prime}, \beta_{k+1}^{\prime}\right), & \left(\alpha_{i}^{\prime \prime}, \alpha_{i+1}^{\prime}\right) \times\left(\beta_{k}^{\prime \prime}, \beta_{k+1}^{\prime}\right), & \left(\alpha_{i+1}^{\prime}, \alpha_{i+1}^{\prime \prime}\right) \times\left(\beta_{k}^{\prime \prime}, \beta_{k+1}^{\prime}\right), \\
\left(\alpha_{i}^{\prime}, \alpha_{i}^{\prime \prime}\right) \times\left(\beta_{k+1}^{\prime}, \beta_{k+1}^{\prime \prime}\right), & \left(\alpha_{i}^{\prime \prime}, \alpha_{i+1}^{\prime}\right) \times\left(\beta_{k+1}^{\prime}, \beta_{k+1}^{\prime \prime}\right), & \left(\alpha_{i+1}^{\prime}, \alpha_{i+1}^{\prime \prime}\right) \times\left(\beta_{k+1}^{\prime}, \beta_{k+1}^{\prime \prime}\right) .
\end{array}
$$

Fixed $i$ and $k$, let us denote by the symbols $\sum q\left(C_{i, k}\right), \sum \widehat{q}\left(C_{i, k}\right)$ the sum of the functions $q, \widehat{q}$ respectively over the eight rectangles $C_{i, k}$ "surrounding" $\left(\alpha_{i}^{\prime \prime}, \alpha_{i+1}^{\prime}\right) \times\left(\beta_{k}^{\prime \prime}, \beta_{k+1}^{\prime}\right)$.

We shall compare both $S(\mathcal{B})$ and $S\left(\mathcal{A}^{\prime \prime}\right)$ with

$$
\sum_{i, k} q\left(\left(\alpha_{i}^{\prime \prime}, \alpha_{i+1}^{\prime}\right) \times\left(\beta_{k}^{\prime \prime}, \beta_{k+1}^{\prime}\right)\right)
$$

( i.e. the "central" elements of the nine above). Indeed, by subadditivity of $q$, and absolute continuity and additivity of the integral $\int \widehat{h}$, we get 


$$
\begin{aligned}
q\left(\left(a_{i}^{\prime \prime}, a_{i+1}^{\prime \prime}\right) \times\left(b_{k}^{\prime \prime}, b_{k+1}^{\prime \prime}\right)\right) & \leq q\left(\left(\alpha_{i}^{\prime \prime}, \alpha_{i+1}^{\prime}\right) \times\left(\beta_{k}^{\prime \prime}, \beta_{k+1}^{\prime}\right)\right)+\sum q\left(C_{i, k}\right) \\
(6) & \leq q\left(\left(\alpha_{i}^{\prime \prime}, \alpha_{i+1}^{\prime}\right) \times\left(\beta_{k}^{\prime \prime}, \beta_{k+1}^{\prime}\right)\right)+\left[\sum \widehat{q}\left(C_{i, k}\right)\right] w \\
& \leq q\left(\left(\alpha_{i}^{\prime \prime}, \alpha_{i+1}^{\prime}\right) \times\left(\beta_{k}^{\prime \prime}, \beta_{k+1}^{\prime}\right)\right)+\frac{\varepsilon}{8(n+3)(l+3)} w .
\end{aligned}
$$

Summing over $i$ and $k$ in (6), and considering also the inequality analogous to (6), obtained by exchanging the roles of $\left(a_{i}^{\prime \prime}, a_{i+1}^{\prime \prime}\right) \times\left(b_{k}^{\prime \prime}, b_{k+1}^{\prime \prime}\right)$ and $\left(\alpha_{i}^{\prime \prime}, \alpha_{i+1}^{\prime}\right) \times\left(\beta_{k}^{\prime \prime}, \beta_{k+1}^{\prime}\right)$, we get

$$
\left|S\left(\mathcal{A}^{\prime \prime}\right)-\sum_{i, k} q\left(\left(\alpha_{i}^{\prime \prime}, \alpha_{i+1}^{\prime}\right) \times\left(\beta_{k}^{\prime \prime}, \beta_{k+1}^{\prime}\right)\right)\right| \leq \frac{\varepsilon}{8} w .
$$

Similarly, we have

$$
S(\mathcal{B}) \geq \sum_{i, k} q\left(\left(\alpha_{i}^{\prime \prime}, \alpha_{i+1}^{\prime}\right) \times\left(\beta_{k}^{\prime \prime}, \beta_{k+1}^{\prime}\right)\right)
$$

and

$$
S(\mathcal{B})-\sum_{i, k} q\left(\left(\alpha_{i}^{\prime \prime}, \alpha_{i+1}^{\prime}\right) \times\left(\beta_{k}^{\prime \prime}, \beta_{k+1}^{\prime}\right)\right) \leq \frac{\varepsilon}{8} w .
$$

Now, it is clear that $\left|S(\mathcal{B})-S\left(\mathcal{A}^{\prime \prime}\right)\right| \leq \frac{\varepsilon}{4} w$. So, we have proved that, for every division $\mathcal{A}$ and $\varepsilon>0$, there exists a cartesian rational division $\mathcal{B}$, such that $S(\mathcal{B}) \geq S(\mathcal{A})-\frac{\varepsilon}{4} w$. This implies that

$$
V(f)=\sup \{S(\mathcal{B}), \mathcal{B} \text { cartesian rational division }\} .
$$

Let us enumerate the family $\left(\mathcal{B}_{n}\right)_{n}$ of all rational cartesian divisions. For each integer $n$, set $p_{n}=V(f)-\sup _{j \leq n} S\left(\mathcal{B}_{j}\right)$. Clearly, $\left(p_{n}\right)_{n}$ is an $(o)$ sequence. Now, define $a_{i, j}=2 p_{i \vee j}$ : then $\left(a_{i, j}\right)_{i, j}$ is a $(D)$-sequence. We shall show that $\left(a_{i, j}\right)_{i, j}$ works to demonstrate that $V(f)=I(q)$, according to (5.1.1). Fix any $\varphi \in \mathbb{N}^{\mathbb{N}}$. Without loss of generality, $\varphi$ can be taken to be increasing, so $\vee_{i=1}^{\infty} a_{i, \varphi(i)}=2 p_{\varphi(1)}$. In correspondence with $\varphi(1)$ take any rational cartesian division $\mathcal{B}_{\varphi}$, finer than $\mathcal{B}_{1}, \mathcal{B}_{2}, \ldots, \mathcal{B}_{\varphi(1)}$. So we have $S\left(\mathcal{B}_{\varphi}\right) \geq \sup _{i \leq \varphi(1)} S\left(\mathcal{B}_{i}\right)=V(f)-p_{\varphi(1)}$, and hence $V(f)-S\left(\mathcal{B}_{\varphi}\right) \leq p_{\varphi(1)}$. Now, if we take two arbitrary divisions $\Pi, \Pi^{\prime}$ finer than $\mathcal{B}_{\varphi}$, we have $S\left(\mathcal{B}_{\varphi}\right) \leq S(\Pi), S\left(\mathcal{B}_{\varphi}\right) \leq S\left(\Pi^{\prime}\right)$, hence $V(f)-S(\Pi) \leq p_{\varphi(1)}, V(f)-S\left(\Pi^{\prime}\right) \leq$ $p_{\varphi(1)}$ and therefore $\left|S(\Pi)-S\left(\Pi^{\prime}\right)\right| \leq 2 p_{\varphi(1)}=\vee_{i=1}^{\infty} a_{i, \varphi(i)}$. This shows (5.2.1), and so $V(f)=I(q)$. 
Theorem 5.4. Let $f$ be an $R$-valued, w-dominated, $K H$-integrable function defined on $J$. Then $|f|$ is $K H$-integrable on $J$ and $\int_{J}|f|=V(f)$.

Proof. Fix arbitrarily $\varepsilon>0$. We saw, at the Step 2 of Lemma 5.3, that there is a regulator $\left(a_{i, j}\right)_{i, j}$, such that to every $\varphi \in \mathbb{N}^{\mathbb{N}}$ there corresponds a (cartesian rational) division $\Pi_{\varphi}=\left\{K_{1}, \ldots, K_{n_{\varphi}}\right\}$ with the property that, for any two divisions $\Pi_{D}, \Pi^{D}$ which are refinements of $\Pi_{\varphi}$, we get

$$
\left|\sum_{L \in \Pi^{D}} q(L)-\sum_{M \in \Pi_{D}} q(M)\right| \leq \bigvee_{i=1}^{\infty} a_{i, \varphi(i)} .
$$

Let now $B_{\varphi}:=\cup_{r=1}^{n_{\varphi}} \partial K_{r}$. We get that $\lambda_{2}\left(B_{\varphi}\right)=0$ and, thanks to $w$ domination and $K H$-integrability of $\widehat{h}$, there exists a gauge $\delta_{B}$ on $J$ such that

$$
\sum_{(L, x) \in \pi_{B}}|f(x)| \lambda_{2}(L) \leq\left(\sum_{(L, x) \in \pi_{B}} \widehat{h}(x) \lambda_{2}(L)\right) w \leq \varepsilon w
$$

for each $\delta_{B}$-fine decomposition $\Pi_{B}$, with tags in $B_{\varphi}$ (see [16, Theorem 6.5.1]).

By the Henstock Lemma (3.5.2) applied to each of the $K_{r}$ 's, by virtue of integrability of $f$ there exists a regulator $\left(\alpha_{i, j}\right)_{i, j}$ (which without loss of generality can be adapted also to $q$ ) such that, in correspondence with $\varphi \in \mathbb{N}^{\mathbb{N}}$ fixed at the beginning of the step, a gauge $\delta_{r}$ on $K_{r}$ can be found, with the property that, for each $\delta_{r}$-fine partition $\Pi_{r}^{*}$ of $K_{r}$,

$$
\sum_{(L, x) \in \Pi_{r}^{*}}\left|f(x) \lambda_{2}(L)-\int_{L} f\right| \leq \frac{1}{n_{\varphi}} \bigvee_{i=1}^{\infty} \alpha_{i, \varphi(i)},
$$

and hence a fortiori

$$
\begin{gathered}
\sum_{(L, x) \in \Pi_{r}^{*}}|f(x)| \lambda_{2}(L)-\sum_{(L, x) \in \Pi_{r}^{*}} q(L) \leq \frac{1}{n_{\varphi}} \bigvee_{i=1}^{\infty} \alpha_{i, \varphi(i)}, \\
\sum_{(L, x) \in \Pi_{r}^{*}} q(L)-\sum_{(L, x) \in \Pi_{r}^{*}}|f(x)| \lambda_{2}(L) \leq \frac{1}{n_{\varphi}} \bigvee_{i=1}^{\infty} \alpha_{i, \varphi(i)},
\end{gathered}
$$

that is

$$
\left|\sum_{(L, x) \in \Pi_{r}^{*}}\right| f(x)\left|\lambda_{2}(L)-\sum_{(L, x) \in \Pi_{r}^{*}} q(L)\right| \leq \frac{1}{n_{\varphi}} \bigvee_{i=1}^{\infty} \alpha_{i, \varphi(i)}
$$


We now construct a gauge $\delta$ on $J$ in the following way. If $x \in B_{\varphi}$, then $x \in \overline{K_{r_{j}}}$ for some $r_{j}$. In this case, put $\delta(x)=\delta_{B}(x) \cap\left(\cap_{r_{j}} \delta_{r_{j}}(x)\right)$, where the last intersection is taken with respect to the $r_{j}$ 's for which $x \in \overline{K_{r_{j}}}$. If $x \in J \backslash B_{\varphi}$, then there is a unique index $j$ such that $x \in K_{j}^{0}$. In this case, set $\delta(x)=\delta_{j}(x) \cap K_{j}^{0}$.

The gauge constructed by this procedure depends of course on $\varphi$. Take any $\delta$-fine partition $\Pi$, and consider $\widetilde{\Pi}:=\Pi \vee \Pi_{\varphi}$, according to the refinement ordering, where $\Pi_{\varphi}$ is the same as at the beginning of the proof. The only rectangles of $\Pi$ that are not rectangles of $\widetilde{\Pi}$ are those for which the tag belongs to $B_{\varphi}$. This allows us to assign to the rectangles of $\widetilde{\Pi}$ the same tags chosen for the rectangles of $\Pi$, thus making $\widetilde{\Pi} \delta$-fine too. Moreover, thanks to (8), we can see that

$$
\left|S_{|f|}(\Pi)-S_{|f|}(\widetilde{\Pi})\right| \leq 2 \varepsilon w,
$$

where $S_{|f|}$ denotes the Riemann sum of $|f|$ along the tagged partitions.

Now, thanks to (9), and adding as $r$ runs from 1 to $n_{\varphi}$, we get

$$
\left|S_{|f|}(\widetilde{\Pi})-S_{q}(\widetilde{\Pi})\right| \leq \bigvee_{i=1}^{\infty} \alpha_{i, \varphi(i)}
$$

where $S_{q}$ means the sum of $q$ along the rectangles of the partition. So, if we take any two $\delta$-fine partitions $\Pi_{1}$ and $\Pi_{2}$, from (10) we have

$$
\left|S_{|f|}\left(\Pi_{1}\right)-S_{|f|}\left(\Pi_{2}\right)\right| \leq\left|S_{|f|}\left(\widetilde{\Pi}_{1}\right)-S_{|f|}\left(\widetilde{\Pi}_{2}\right)\right|+4 \varepsilon w .
$$

Finally, thanks to (7) and (11), we deduce

$$
\begin{aligned}
\left|S_{|f|}\left(\Pi_{1}\right)-S_{|f|}\left(\Pi_{2}\right)\right| & \leq\left|S_{q}\left(\widetilde{\Pi}_{1}\right)-S_{q}\left(\widetilde{\Pi}_{2}\right)\right|+2 \bigvee_{i=1}^{\infty} \alpha_{i, \varphi(i)}+4 \varepsilon w \\
& \leq \bigvee_{i=1}^{\infty} a_{i, \varphi(i)}+2 \bigvee_{i=1}^{\infty} \alpha_{i, \varphi(i)}+4 \varepsilon w
\end{aligned}
$$

for all $\delta$-fine partitions $\Pi_{1}$ and $\Pi_{2}$. Thanks to arbitrariness of $\varepsilon$, we obtain that $|f|$ satisfies the Bolzano-Cauchy condition (3.3.1), and thus $|f|$ is $K H$ integrable.

Finally, let us prove that $\int_{J}|f|=V(f)$. In order to do this, first of all consider

$$
V(f ; \gamma)=\sup \left\{\sum_{i=1}^{n} q\left(Y_{i}\right):\left(Y_{i}, \eta_{i}\right)_{i=1}^{n} \gamma \text {-fine and belongs to } \mathcal{C}_{c r}(J)\right\},
$$


where $\gamma$ is a fixed gauge. It is not difficult to check that $V(f)=V(f ; \gamma)$. Let now $I=\int_{J}|f|$. From the definition of $K H$-integral we get the existence of a regulator $\left(c_{i, j}\right)_{i, j}$ with the property that for all $\varphi \in \mathbb{N}^{\mathbb{N}}$ there is a gauge $\gamma$ on $J$ with

$$
I-\bigvee_{i=1}^{\infty} c_{i, \varphi(i)} \leq \sum_{(L, x) \in \Pi}|f(x)| \lambda_{2}(L)
$$

whenever $\Pi$ is a $\gamma$-fine partition of $(a, b)$. Moreover, let $\left(\alpha_{i, j}\right)_{i, j}$ be a regulator associated with $K H$-integrability of $f$ and the Henstock Lemma. Fix now arbitrarily $\varepsilon>0$ and $\varphi \in \mathbb{N}^{\mathbb{N}}$, and let $\gamma^{*}(x)=\gamma(x) \cap \delta(x)$, $x \in(a, b)$, where $\delta$ is the above defined gauge. For each $\gamma^{*}$-fine partition $\Pi$ of $(a, b)$ let $\widetilde{\Pi}:=\Pi \vee \Pi_{\varphi}$, where $\Pi_{\varphi}$ is as at the beginning of the proof. From (8) and (11), by "splitting" the sums on $\Pi$ along the intervals with tags in and outside $B_{\varphi}$ respectively, we have:

$$
\begin{aligned}
I-\bigvee_{i=1}^{\infty} c_{i, \varphi(i)} & \leq \sum_{(L, x) \in \Pi}|f(x)| \lambda_{2}(L) \leq \varepsilon w+\sum_{(L, x) \in \widetilde{\Pi}}|f(x)| \lambda_{2}(L) \\
& \leq \sum_{(L, x) \in \widetilde{\Pi}} q(L)+\bigvee_{i=1}^{\infty} \alpha_{i, \varphi(i)}+\varepsilon w \leq V(f)+\bigvee_{i=1}^{\infty} \alpha_{i, \varphi(i)}+\varepsilon w .
\end{aligned}
$$

Thus, by arbitrariness of $\varepsilon$ and $\varphi$ and weak $\sigma$-distributivity of $R$, we obtain $I \leq V(f)$.

Concerning the converse inequality, let $\left(d_{i, j}\right)_{i, j}$ be a regulator related with $K H$-integrability of $|f|$, fix arbitrarily $\varphi \in \mathbb{N}^{\mathbb{N}}$ and let $\gamma=\gamma(\varphi)$ be a corresponding gauge on $J$ according to $K H$-integrability of $|f|$. We have:

$$
V(f)=V(f ; \gamma) \leq \sup \sum_{p=1}^{k}\left|f\left(\tau_{p}\right)\right| \lambda_{2}\left(K_{p}\right) \leq I+\bigvee_{i=1}^{\infty} d_{i, \varphi(i)}
$$

(Here the supremum is taken with respect to all $\gamma$-fine cartesian rational partitions). So, by arbitrariness of $\varphi$ and weak $\sigma$-distributivity of $R$ again, we obtain $V(f) \leq I$. This concludes the proof. 


\section{References}

[1] S. J. Bernau, Unique representation of Archimedean lattice groups and normal Archimedean lattice rings, Proc. London Math. Soc., 15 (1965), 599-631.

[2] A. Boccuto, Differential and Integral Calculus in Riesz Spaces, Tatra Mountains Math. Publ., 14 (1998), 293-323.

[3] A. Boccuto and B. Riečan, On the Henstock-Kurzweil integral for Rieszspace-valued functions defined on unbounded intervals, Czech. Math. J., 54 (129) (2004), 591-607.

[4] A. Boccuto and B. Riečan, The Kurzweil-Henstock Integral for Riesz Space-Valued Maps Defined in Abstract Topological Spaces and Convergence Theorems, Pan American Math. J., 16 (2006), 63-79.

[5] A. Boccuto, B. Riečan and A. R. Sambucini, Convergence and Fubini theorems for metric semigroup-valued functions defined on unbounded rectangles, Pan American Math. J., 19 (2009), 1-12.

[6] B. Bongiorno, The Henstock-Kurzweil Integral, Handbook of Measure Theory, North-Holland, Amsterdam, 2002, 587-615.

[7] B. Bongiorno, L. Di Piazza and K. Musiał, Kurzweil-Henstock and Kurzweil-Henstock-Pettis integrability of strongly measurable functions, Math. Bohem., 131 (2006), 211-223.

[8] D. Candeloro, Riemann-Stieltjes Integration in Riesz Spaces, Rend. Mat. (Roma), 16 (1996), 563-585.

[9] L. Cesari, Quasi-additive set functions and the concept of integral over a variety, Trans. Amer. Math. Soc., 102 (1962), 94-113.

[10] K. Ciesielski and M. Laczkovich, Strong Fubini properties for measure and category, Fund. Math., 178 (2003), 171-188.

[11] R. O. Davies and Z. Schuss, A proof that Henstock's integral includes Lebesgue's, J. London Math. Soc., 2 (1970), 561-562.

[12] M. Duchoň and B. Riečan, On the Kurzweil-Stieltjes integral in ordered spaces, Tatra Mountains Math. Publ., 8 (1996), 133-141.

[13] D. H. Fremlin, Topological Riesz Spaces and Measure Theory, Cambridge Univ. Press, 1974.

[14] R. Henstock, A problem in two-dimensional integration, J. Austral. Math. Soc. Ser. A, 35 (1983), 386-404.

[15] J. Kurzwell, Nichtabsolut Konvergente Integrale, Teubner, Leipzig, 1980. 
[16] P. Y. Lee and R. Výborný, The integral: An Easy Approach After Kurzweil and Henstock, Cambridge Univ. Press, 2000.

[17] T. Y. Lee, A full descriptive definition of the Henstock-Kurzweil integral in the Euclidean space, Proc. London Math. Soc., 87 (2003), 677-700.

[18] T. Y. Lee, Product variational measures and Fubini-Tonelli type theorems for the Henstock-Kurzweil integral, J. Math. Anal. Appl., 298 (2004), 677-692.

[19] T. Y. Lee, Product variational measures and Fubini-Tonelli type theorems for the Henstock-Kurzweil integral II, J. Math. Anal. Appl., 323 (2006), 741-745.

[20] W. A. J. Luxemburg and A. C. Zaanen, Riesz Spaces, I, North-Holland Publishing Co., 1971.

[21] P. Mcgill, Integration in vector lattices, J. London Math. Soc., 11 (1975), 347-360.

[22] E. J. Mcshane, A Riemann-type integral that includes LebesgueStieltjes, Bochner and stochastic integrals, Mem. Amer. Math. Soc., 88 (1969).

[23] W. Pfeffer, The Riemann Approach to Integration, Cambridge Univ. Press, Cambridge, 1993.

[24] B. Riečan and T. Neubrunn, Integral, Measure and Ordering, Kluwer Academic Publishers/Ister Science, Bratislava, 1997.

[25] C. Vinti, L'integrale di Fubini-Tonelli nel senso di Weierstrass. II. Caso ordinario, Annali Scuola Normale Sup. Pisa, 22 (1968), 355-376.

[26] M. Vráblelová, Fubini's Theorem in ordered spaces, Acta Math. (Nitra), 5 (2002), 37-42.

[27] M. Vráblelová, Double Kurzweil-Henstock integral in Riesz spaces, Acta Math. (Nitra), 7 (2004), 45-52.

[28] M. Vráblelová, Fubini's theorem in metric semigroups, Acta Math. (Nitra), 8 (2005), 31-37.

[29] M. Wright, Stone-algebra-valued measures and integrals, Proc. London Math. Soc., 19 (1969), 107-122. 
Dipartimento di Matematica e Informatica

via Vanvitelli,1, I-06123 PERUGIA

Italy

(E-mail : boccuto@dmi.unipg.it)

(E-mail : candelor@dmi.unipg.it)

(E-mail : matears1@unipg.it) 


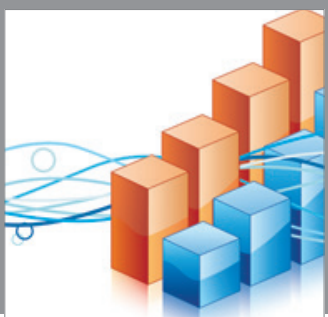

Advances in

Operations Research

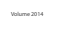

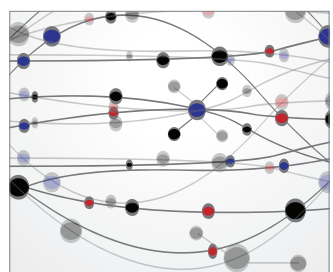

\section{The Scientific} World Journal
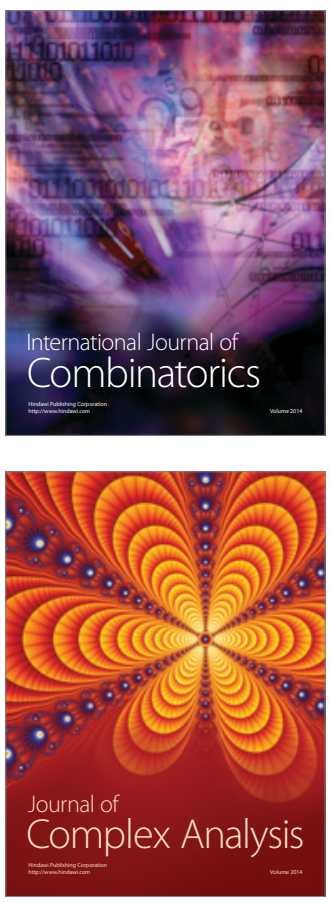

International Journal of

Mathematics and

Mathematical

Sciences
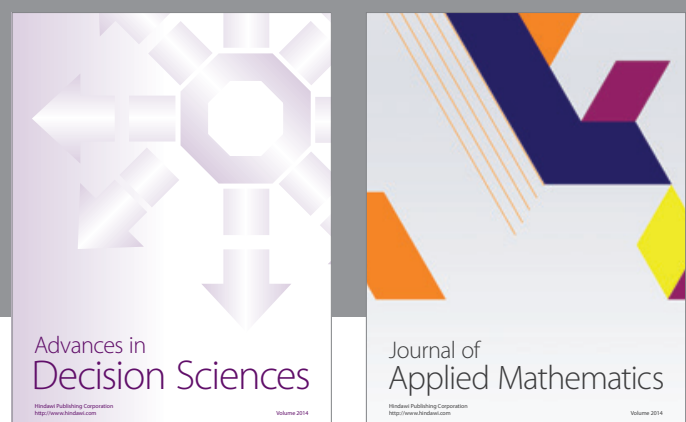

Journal of

Applied Mathematics
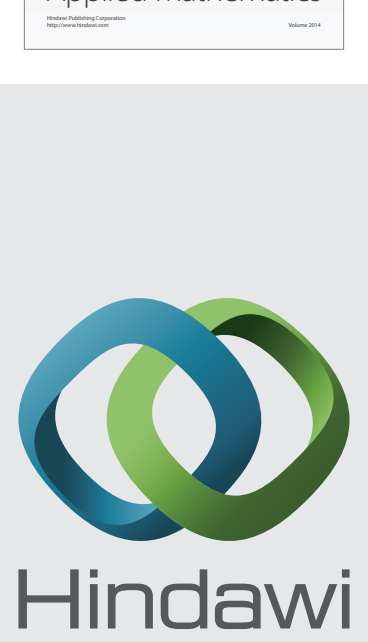

Submit your manuscripts at http://www.hindawi.com
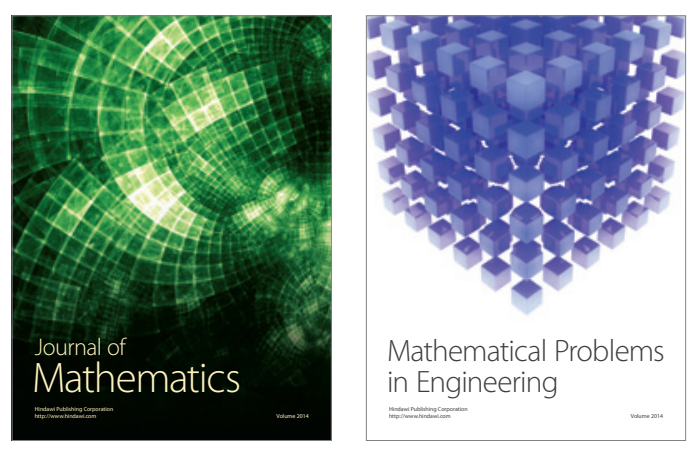

Mathematical Problems in Engineering
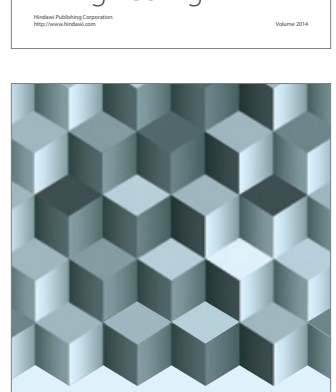

Journal of

Function Spaces
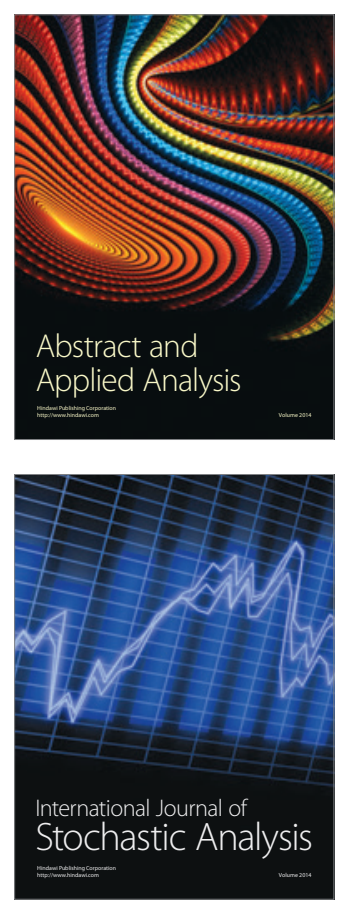

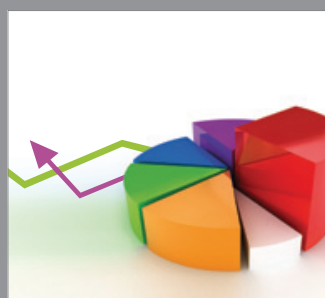

ournal of

Probability and Statistics

Promensencen
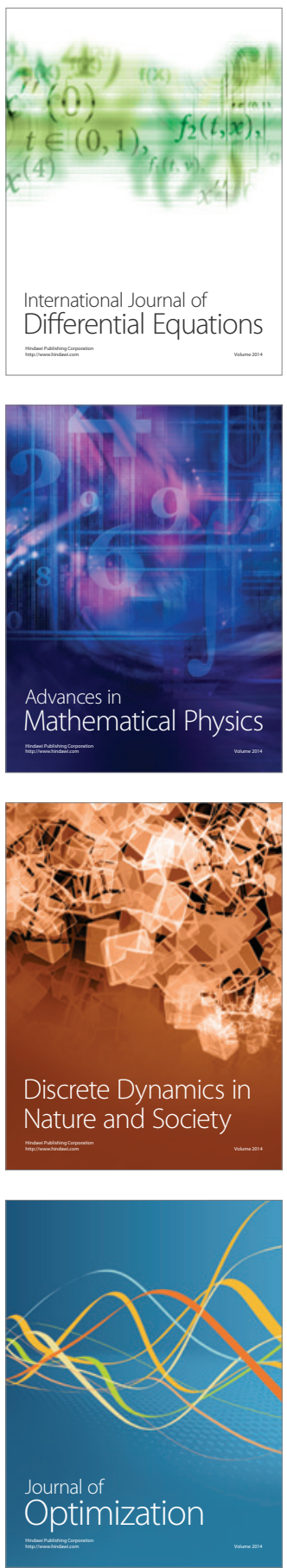